Ther mal conduct ivity of ur ani um di oxi de by nonequi I i bri um Nol ecul ar dynamics si mul at i on

\begin{tabular}{|l|l|}
\hline 著者 & $\begin{array}{l}\text { Not oyana Sat oshi, I chi kawa Yasushi, H wat ar i } \\
\text { Yasuaki, Oe Aki r a }\end{array}$ \\
\hline $\begin{array}{l}\text { j our nal or } \\
\text { publ i cat i on t i t l e }\end{array}$ & Physi cal Revi ew B \\
\hline vol ume & 60 \\
\hline number & 1 \\
\hline page $r$ ange & 292-298 \\
\hline year & 1999- 01- 01 \\
\hline URL & ht t p: //hdl . handl e. net /2297/1674 \\
\hline
\end{tabular}




\title{
Thermal conductivity of uranium dioxide by nonequilibrium molecular dynamics simulation
}

\author{
Satoshi Motoyama \\ Department of Energy Technology, The Japan Research Institute, Limited, Osaka, 550-0013, Japan \\ Yasushi Ichikawa and Yasuaki Hiwatari \\ Department of Computational Science, Faculty of Science, Kanazawa University, Kanazawa, 920-1164, Japan \\ Akira Oe \\ Department of Fuel Engineering, PWR Fuel Division, Nuclear Fuel Industries, Ltd., Osaka, 590-0451, Japan
}

(Received 9 April 1998; revised manuscript received 19 January 1999)

\begin{abstract}
The thermal conductivity of $\mathrm{UO}_{2}$ pellet, which is used as nuclear fuel, was calculated using the nonequilibrium classical molecular dynamics method by Evans and Morriss. The result of calculation from 300-3000 $\mathrm{K}$ obtained presents the same tendency as experiments below $2400 \mathrm{~K}$. Above $2400 \mathrm{~K}$, a different tendency was observed in which the electron conduction was not negligible. The thermal conductivity using the nonequilibrium molecular dynamics method was calculated with about 1/10 number of simulation steps compared with the equilibrium molecular dynamics simulation. It was found that the nonequilibrium molecular dynamics simulation is quite effective. [S0163-1829(99)04925-5]
\end{abstract}

\section{INTRODUCTION}

The thermal conductivity of nuclear fuel pellets is an important physical property for the temperature analysis in nuclear reactors. Recently it has become an especially important subject to make clear the mechanism of a fall of the thermal conductivity in a high burnup fuel and mixed-oxide (MOX) fuel which is presently planned to be used in a light water reactor.

About the former, the reason has been explained by the fact that FP (fission product) was accumulated into the fuel pellet, or the defect (vacancy or interstitial) was increased by irradiation. But it has not yet been resolved. For the latter, the thermal conductivity of MOX pellet is being evaluated smaller compared with a $\mathrm{UO}_{2}$ pellet. However, the physical basis and the theoretical mechanism of the fall of thermal conductivity are still not clear. There are such few data in higher temperatures than $1800 \mathrm{~K}$. In such a high-temperature region, the thermal conductivity of MOX pellet may not be in the same behavior as that of a $\mathrm{UO}_{2}$ pellet.

Thermal conductivity has already been calculated for the $L-J$ crystal with Kubo formula. And the formalism of thermal conductivity of disordered harmonic solids was implemented in Ref. 1. The formalism will become very important for us to calculate the thermal conductivity of MOX pellet at the high-temperature region. The present work is to clarify the characteristic of important physical properties as thermal conductivity and thermal expansion by using molecular dynamics simulation. However, since in Coulomb systems the time correlation function of energy currents shows an oscillatory behavior and keeps its memory for a long time, the calculation of the thermal conductivity with a high accuracy is very difficult. Then, in this work, we adopt the nonequilibrium molecular dynamics simulation (NEMD) by Evans and Morriss by adding the external force in the equations of motion to each particle.

First, we have compared the thermal conductivity of solid
Ar, which is a typical $L-J$ crystal calculated by the equilibrium molecular dynamics simulation, with the result of using nonequilibrium molecular dynamics simulation.

Second, the nonequilibrium molecular dynamics simulation was applied to a Coulomb system, and the thermal conductivity of the $\mathrm{UO}_{2}$ pellet was evaluated.

\section{SIMULATION ALGORITHM OF NEMD}

The method of the nonequilibrium molecular dynamics simulation (NEMD) of Evans and Morriss uses the external force to each particle and the movement of a particle is solved by the equations of motion, 2,3

$$
\dot{\mathbf{r}}_{\mathbf{i}}=\frac{\mathbf{p}_{\mathbf{i}}}{m_{i}}+\overleftrightarrow{\mathbf{C}}_{\mathbf{i}} \cdot \mathbf{F}_{\mathbf{e x t}}(t)
$$

and

$$
\dot{\mathbf{p}}_{\mathbf{i}}=\mathbf{F}_{\mathbf{i}}+\overleftrightarrow{\mathbf{D}}_{\mathbf{i}} \cdot \mathbf{F}_{\text {ext }}(t)-\alpha \mathbf{p}_{\mathbf{i}},
$$

where $\overleftrightarrow{\mathbf{C}}_{\mathbf{i}}$ and $\overleftrightarrow{\mathbf{D}}_{\mathbf{i}}$ are parameters, which should be set for needs of physical quantity under consideration. $\alpha$ is a control parameter which keeps system temperature constant, and it can be determined by the time derivative of the kinetic energy and setting it equal to zero:

$$
\begin{gathered}
\frac{\partial}{\partial t} \sum_{i} \frac{1}{2} m_{i} \dot{\mathbf{r}}_{\mathbf{i}}^{2}=\sum_{i} \dot{\mathbf{r}}_{\mathbf{i}} \cdot \dot{\mathbf{p}}_{\mathbf{i}}=\sum_{i} \dot{\mathbf{r}}_{\mathbf{i}} \cdot\left(\mathbf{F}_{\mathbf{i}}+\overleftrightarrow{\mathbf{D}}_{\mathbf{i}} \cdot \mathbf{F}_{\mathbf{e x t}}(t)-\alpha \mathbf{p}_{\mathbf{i}}\right)=0 \\
\alpha=\frac{\sum_{i}\left[\dot{\mathbf{r}}_{\mathbf{i}} \cdot \mathbf{F}_{\mathbf{i}}+\dot{\mathbf{r}}_{\mathbf{i}} \cdot\left(\overleftrightarrow{\mathbf{D}}_{\mathbf{i}} \cdot \mathbf{F}_{\mathbf{e x t}}(t)\right)\right]}{\sum_{i} \dot{\mathbf{r}}_{\mathbf{i}} \cdot \mathbf{p}_{\mathbf{i}}}
\end{gathered}
$$

We now show how to determine $\overleftrightarrow{\mathbf{C}}_{\mathbf{i}}$ and $\overleftrightarrow{\mathbf{D}}_{\mathbf{i}}$ parameters below in the case of $\alpha=0$. In the linear response theory, Eq. 
(5) is obtained for an arbitrary physical observable $B\left(\left\{r_{i}, p_{i}\right\}_{i=1, \cdots, N}\right)$ using $H_{0}$ (Hamiltonian of the internal energy with no external force): ${ }^{4,5}$

$$
\langle B\rangle_{t}=\langle B\rangle_{e q}+\frac{1}{k_{B} T} \int_{t_{\text {on }}}^{t} d s\left\langle B(t) \dot{H}_{0}(0)\right\rangle
$$

where

$$
H_{0} \equiv \sum_{i=1}^{N}\left[\frac{\left|\mathbf{p}_{\mathbf{i}}\right|^{2}}{2 m_{i}}+\phi\left(\mathbf{r}_{\mathbf{i}}\right)\right]
$$

$\langle B\rangle_{t}$ and $\langle B\rangle_{e q}$ indicate an average value of $B$ at time $t(t$ $\left.\geqslant t_{o n}\right)$, an average value of $B$ before $t_{o n}$, respectively. The time derivative of Eq. (6) becomes

$$
\dot{H}_{0}=\sum_{i=1}^{N}\left[\nabla_{p_{i}} H_{0} \cdot \dot{\mathbf{p}}_{\mathbf{i}}+\nabla H_{0} \cdot \dot{\mathbf{r}}_{\mathbf{i}}\right]=\sum_{i=1}^{N}\left[\frac{\mathbf{p}_{\mathbf{i}} \cdot \dot{\mathbf{p}}_{\mathbf{i}}}{m_{i}}-\mathbf{F}_{\mathbf{i}} \cdot \dot{\mathbf{r}}_{\mathbf{i}}\right] .
$$

When Eqs. (1) and (2) are substituted into Eq. (7), we obtain

$$
\begin{aligned}
\dot{H}_{0} & =\sum_{i=1}^{N}\left[\frac{\mathbf{p}_{\mathbf{i}} \cdot\left(\mathbf{F}_{\mathbf{i}}+\overleftrightarrow{\mathbf{D}}_{\mathbf{i}} \cdot \mathbf{F}_{\text {ext }}\right)}{m_{i}}-\mathbf{F}_{\mathbf{i}} \cdot\left(\frac{\mathbf{P}_{\mathbf{i}}}{m_{i}}+\overleftrightarrow{\mathbf{C}}_{\mathbf{i}} \cdot \mathbf{F}_{\mathbf{e x t}}(t)\right)\right] \\
& =\sum_{i=1}^{N}\left[\frac{\mathbf{p}_{\mathbf{i}} \cdot\left(\overleftrightarrow{\mathbf{D}}_{\mathbf{i}} \cdot \mathbf{F}_{\text {ext }}\right)}{m_{i}}-\mathbf{F}_{\mathbf{i}} \cdot\left(\overleftrightarrow{\mathbf{C}}_{\mathbf{i}} \cdot \mathbf{F}_{\text {ext }}(t)\right)\right]
\end{aligned}
$$

Hereafter $\overleftrightarrow{\mathbf{C}}_{\mathbf{i}}$ is set equal to zero,

$$
\overleftrightarrow{\mathbf{C}}_{\mathbf{i}}=\mathbf{0}
$$

Thus we obtain

$$
\dot{H}_{0}=\sum_{i=1}^{N} \frac{\mathbf{p}_{\mathbf{i}} \cdot\left(\overleftrightarrow{\mathbf{D}}_{\mathbf{i}} \cdot \mathbf{F}_{\mathbf{e x t}}(t)\right)}{m_{i}}
$$

$\overleftrightarrow{\mathbf{D}}_{\mathbf{i}}$ is defined so as to satisfy Eq. (11), where $\mathbf{J}_{\mathbf{k}=\mathbf{0}}^{\mathbf{e}}$ is the energy current.

$$
\dot{H}_{0}=\sum_{i=1}^{N} \frac{\mathbf{p}_{\mathbf{i}} \cdot\left(\overleftrightarrow{\mathbf{D}}_{\mathbf{i}} \cdot \mathbf{F}_{\mathbf{e x t}}(t)\right)}{m_{i}} \equiv \mathbf{J}_{\mathbf{k}=\mathbf{0}}^{\mathbf{e}} \cdot \mathbf{F}_{\mathbf{e x t}}(t) .
$$

Using these $\overleftrightarrow{\mathbf{C}}_{\mathbf{i}}$ and $\overleftrightarrow{\mathbf{D}}_{\mathbf{i}}$, we can obtain the formula for the thermal conductivity. By substituting

$$
B=\mathbf{J}_{\mathbf{k}=\mathbf{0}}^{\mathbf{e}}
$$

and

$$
\dot{H}_{0}=\mathbf{J}_{\mathbf{k}=\mathbf{0}}^{\mathbf{e}} \cdot \mathbf{F}_{\mathbf{e x t}}(t)
$$

into Eq. (5),

$$
\left\langle\mathbf{J}_{\mathbf{k}=\mathbf{0}}^{\mathbf{e}}\right\rangle_{t}=\left\langle\mathbf{J}_{\mathbf{k}=\mathbf{0}}^{\mathbf{e}}\right\rangle_{e q}+\frac{1}{k_{B} T} \int_{t_{\text {on }}}^{t} d s\left\langle\mathbf{J}_{\mathbf{k}=\mathbf{0}}^{\mathbf{e}}(t)\left(\mathbf{J}_{\mathbf{k}=\mathbf{0}}^{\mathbf{e}} \cdot \mathbf{F}_{\mathbf{e x t}}(s)\right)\right\rangle .
$$

If in Eq. (14) the external force is set only to one direction, we obtain the energy current for that direction:

$$
\left\langle J_{k=0}^{e}\right\rangle_{t}=\left\langle J_{k=0}^{e}\right\rangle_{e q}+\frac{F_{\text {ext }}}{k_{B} T} \int_{t_{\text {on }}}^{t} d s\left\langle J_{k=0}^{e}(t) J_{k=0}^{e}(s)\right\rangle .
$$

Since the average of the energy current in the equilibrium state which does not contain the external force is equal to zero, Eq. (15) becomes

$$
\left\langle J_{k=0}^{e}\right\rangle_{t}=\frac{F_{\text {ext }}}{k_{B} T} \int_{t_{\text {on }}}^{t} d s\left\langle J_{k=0}^{e}(t) J_{k=0}^{e}(0)\right\rangle .
$$

On the other hand, with the Kubo formula ${ }^{7}$ the thermal conductivity $\lambda$ is given by

$$
\lambda=\frac{1}{V k_{B} T^{2}} \int_{0}^{\infty} d s\left\langle J_{k=0}^{e}(s) J_{k=0}^{e}(0)\right\rangle .
$$

From Eqs. (16) and (17), we obtain $\lambda$ :

$$
\lambda=\lim _{t \rightarrow \infty} \frac{1}{V T F_{\text {ext }}}\left\langle J_{k=0}^{e}\right\rangle_{t} .
$$

Using the heat flux $\psi\left(F_{\text {ext }}\right)$ which is defined by

$$
\psi\left(F_{\text {ext }}\right)=\lim _{t \rightarrow \infty} \frac{1}{V T}\left\langle J_{k=0}^{e}\right\rangle_{t},
$$

we obtain a linear relation between $F_{\text {ext }}$ and $\psi\left(F_{\text {ext }}\right)$ with a coefficient $\lambda$ :

$$
\psi\left(F_{\text {ext }}\right)=\lambda F_{\text {ext }}
$$

We calculate the heat flux with various $F_{\text {ext }}$, and $\lambda$ is obtained by the slope of the fitting line of $F_{\text {ext }}$ and $\psi\left(F_{\text {ext }}\right)$.

\section{NUMERICAL CALCULATION OF THERMAL CONDUCTIVITY}

\section{A. Argon}

\section{Equilibrium molecular dynamics in Ar}

We have carried out an equilibrium molecular dynamics simulation with usual equations of motion:

$$
\dot{\mathbf{r}}_{\mathbf{i}}=\frac{\mathbf{p}_{\mathbf{i}}}{m_{i}}
$$

and

$$
\dot{\mathbf{p}}_{\mathbf{i}}=\mathbf{F}_{\mathbf{i}} \text {. }
$$

Here, we used the following model potential for Ar. ${ }^{6}$

$$
\phi\left(r_{i j}\right)=4 \epsilon\left[\left(\frac{\sigma}{\left|\mathbf{r}_{\mathbf{i j}}\right|}\right)^{12}-\left(\frac{\sigma}{\left|\mathbf{r}_{\mathbf{i j}}\right|}\right)^{6}\right],
$$

where

$$
\sigma=3.405(\AA)
$$

and

$$
\epsilon=119.8(\mathrm{~K}) \text {. }
$$

The calculation conditions of the equilibrium molecular dynamics simulation are number of particles, 256; fcc lattice constant, $5.3091 \AA$ A; total simulation steps, 100 000; unit time step, 5.0 fsec; algorithm, Gear (6 value 1st order); temperature, $72.875 \mathrm{~K}$. The energy current $\mathbf{J}(t)$ is given by the following equation: ${ }^{7}$ 


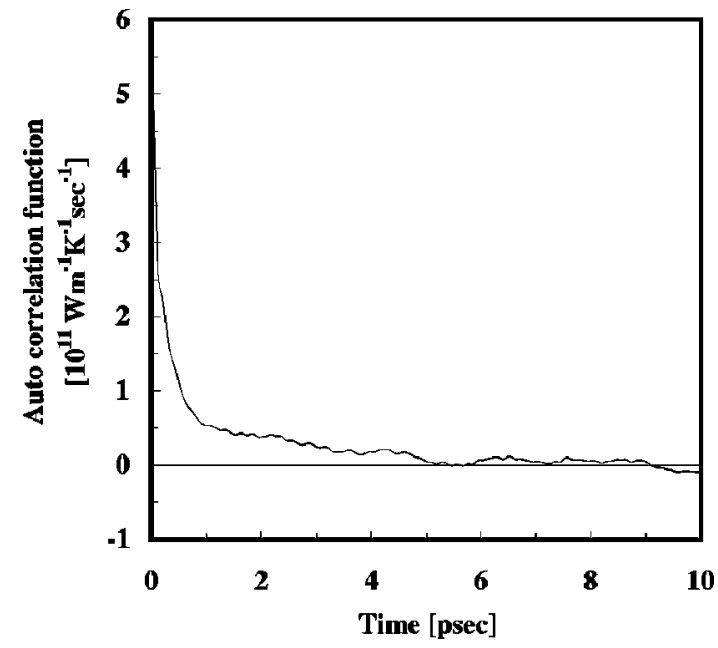

FIG. 1. Autocorrelation function in Ar-EMD calculation.

$$
\mathbf{J}(t)=\sum_{i=1}^{N} \dot{\mathbf{r}}_{\mathbf{i}}\left[\frac{\left|\mathbf{p}_{\mathbf{i}}\right|^{2}}{m_{i}}+\Phi\left(\mathbf{r}_{\mathbf{i}}\right)\right]+\frac{1}{2} \sum_{i=0}^{N} \sum_{j=0}^{N}\left(\dot{\mathbf{r}}_{\mathbf{i}} \cdot \mathbf{r}_{\mathbf{i}}\right) \mathbf{F}_{\mathbf{i j}}
$$

where the minimum image condition was used. Then, the instantaneous thermal conductivity $\lambda(t)$ at time $t$ is obtained by

$$
\lambda(t)=\frac{1}{V k_{B T}^{2}} \int_{0}^{t} d s\langle\mathbf{J}(s) \mathbf{J}(0)\rangle .
$$

We show the autocorrelation function of the energy current $1 / V k_{B} T^{2}\langle\mathbf{J}(s) \mathbf{J}(0)\rangle$ in Fig. 1, and the integral of the autocorrelation function in Fig. 2. From Fig. 2, the thermal conductivity $\lambda$ at $72.9 \mathrm{~K}$ for $\mathrm{Ar}$ is estimated to be 0.286 $\mathrm{W} \mathrm{m}{ }^{-1} \mathrm{~K}^{-1}$, which is also shown in Fig. 4.

\section{Nonequilibrium molecular dynamics in Ar}

In the nonequilibrium molecular dynamics method, we set $\overleftrightarrow{\mathbf{D}}_{\mathbf{i}}$ as

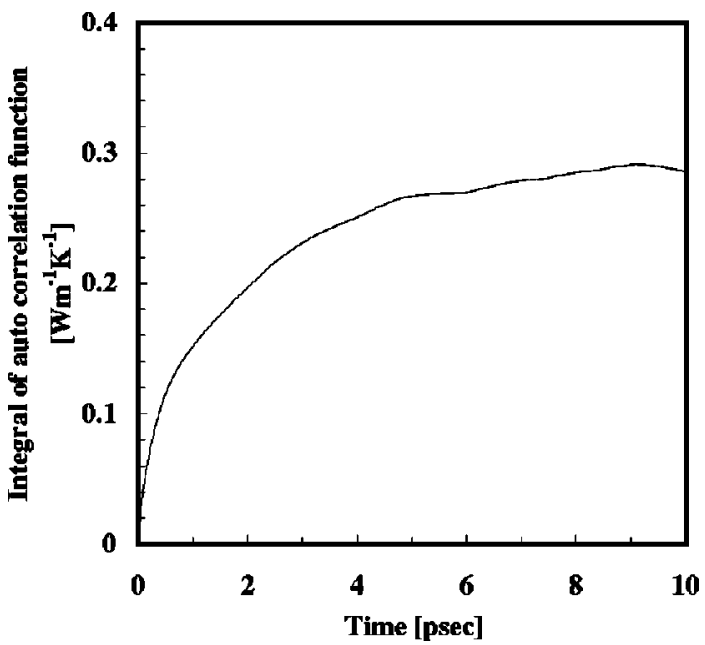

FIG. 2. Integral of autocorrelation function in Ar-EMD calculation.

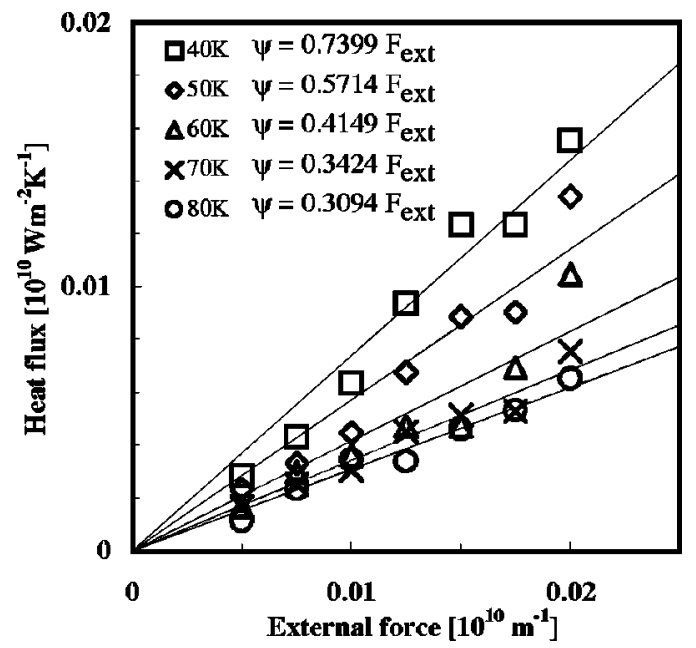

FIG. 3. Heat fluxes as a function of the external force in ArNEMD calculation.

$$
\overleftrightarrow{\mathbf{D}}_{\mathbf{i}}=\overleftrightarrow{\mathbf{D}}_{\mathbf{i}}^{*}-\frac{1}{N} \sum_{j=0}^{N} \overleftrightarrow{\mathbf{D}}_{\mathbf{j}}^{*}
$$

and

$$
\begin{aligned}
& \overleftrightarrow{\mathbf{D}}_{\mathbf{i}}^{*}=\left[\frac{1}{2} m_{i} \dot{\mathbf{r}}_{\mathbf{i}}^{2}+\frac{1}{2} \sum_{j \neq i}^{N} \phi\left(r_{i j}\right)\right]\left(\begin{array}{ccc}
1 & 0 & 0 \\
0 & 1 & 0 \\
0 & 0 & 1
\end{array}\right) \\
& -\left(\begin{array}{ccc}
\frac{1}{2} \sum_{j=1}^{N} x_{i j} F_{x, i j} & 0 & 0 \\
0 & \frac{1}{2} \sum_{j=1}^{N} y_{i j} F_{y, i j} & 0 \\
0 & 0 & \frac{1}{2} \sum_{j=1}^{N} z_{i j} F_{z, i j}
\end{array}\right)
\end{aligned}
$$

The conditions of the nonequilibrium molecular dynamics simulation are $^{6}$ number of particles, 500; fcc lattice constant, $5.3091 \AA$; total simulation steps, 10000 , unit time step, 5.0 fsec; algorithm, Gear (6 value 1st order); temperature, 40, $50,60,70$, and $80 \mathrm{~K}$.

We show the relation between $F_{\text {ext }}$ and $\psi\left(F_{\text {ext }}\right)$ obtained by the nonequilibrium molecular dynamics calculation in Fig. 3. The straight lines in Fig. 3 are obtained by leastsquare fitting of $F_{\text {ext }}$ and $\psi\left(F_{\text {ext }}\right)$ at each temperature. Thus we have obtained the thermal conductivity by the present molecular dynamics calculation, and show them in Fig. 4 with some experiments. 8,9

\section{B. Uranium dioxide}

\section{Equilibrium molecular dynamics in $\mathrm{UO}_{2}$}

We have used the $\mathrm{UO}_{2}$ model potential fitted by J.R. Walker and C.R.A. Catlow, which is essentially of the BornMayer or Buckingham formula. We have also used Coulomb potentials in addition to this short-distance potential: ${ }^{10,11}$ 


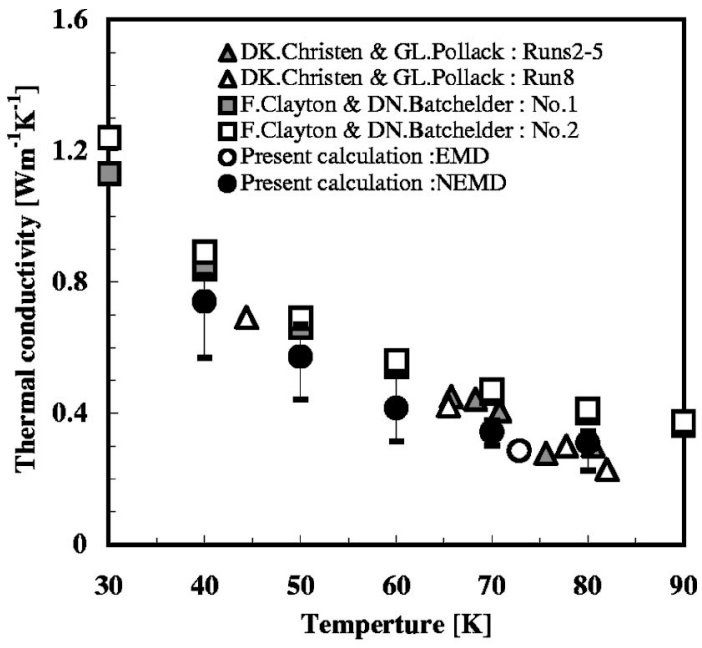

FIG. 4. Thermal conductivity of Ar.

$$
\phi\left(r_{i j}\right)=\frac{e^{2}}{4 \pi \epsilon_{0}} \frac{Z_{i} Z_{j}}{r_{i j}}+A_{i j} e^{-r_{i j} / B_{i j}}-\frac{C_{i j}}{r_{i j}{ }^{6}}
$$

In Table I, potential parameters used in these works were listed. In the computer simulation of Coulomb forces, we have used the method of Ewald as usual.

For Coulomb potentials, the equation of the energy current $\mathbf{J}(t)$ is expressed by ${ }^{4}$

$$
\begin{aligned}
\mathbf{J}(t)= & \sum_{i=1}^{N}\left[\frac{\left|\mathbf{p}_{\mathbf{i}}\right|^{2}}{2 m_{i}}+\Phi\left(\mathbf{r}_{\mathbf{i}}\right)\right] \dot{\mathbf{r}}_{\mathbf{i}} \\
& +\frac{1}{4 \pi \epsilon_{0}} \sum_{i=1}^{N} \dot{\mathbf{r}}_{\mathbf{i}}\left(\begin{array}{ccc}
S_{x x} & S_{y x} & S_{z x} \\
S_{x y} & S_{y y} & S_{z y} \\
S_{x z} & S_{y z} & S_{z z}
\end{array}\right),
\end{aligned}
$$

where

$$
\begin{aligned}
S_{a b}= & \frac{1}{2} \sum_{j \neq i}^{N} \frac{a_{i j} b_{i j}}{\left|\mathbf{r}_{\mathbf{i j}}\right|^{2}}\left[Z_{i} Z_{j}\left(\frac{\operatorname{erfc}\left(\kappa\left|\mathbf{r}_{\mathbf{i j}}\right|\right)}{\left|\mathbf{r}_{\mathbf{i j}}\right|}+\frac{2 \kappa}{\sqrt{\pi}} e^{-\kappa^{2}\left|\mathbf{r}_{\mathbf{i j}}\right|}\right)\right. \\
& \left.+\frac{A_{i j}\left|\mathbf{r}_{\mathbf{i j}}\right|}{B_{i j}} e^{-\left|\mathbf{r}_{\mathbf{i j}}\right| / B_{i j}}-\frac{6 C_{i j}}{\left|\mathbf{r}_{\mathbf{i j}}\right|^{6}}\right] \\
& -Z_{i} \sum_{\mathbf{h}} \frac{h_{a}}{|\mathbf{h}|} \frac{h_{b}}{|\mathbf{h}|} \frac{e^{-\pi^{2}|\mathbf{h}|^{2} / \kappa^{2}}}{\pi|\mathbf{h}|^{2}}\left(1+\frac{\pi^{2}|\mathbf{h}|^{2}}{\kappa^{2}}\right)
\end{aligned}
$$

TABLE I. Potential parameters of $\mathrm{UO}_{2}$.

\begin{tabular}{lclc}
\hline \hline$i-j$ & $A_{i j}[\mathrm{eV}]$ & $B_{i j}[\AA]$ & $C_{i j}\left[\mathrm{eV} \AA^{6}\right]$ \\
\hline $\mathrm{O}^{2-}-\mathrm{O}^{2-}$ & 50259.33984 & 0.15285 & 72.65339 \\
$\mathrm{U}^{4+}-\mathrm{O}^{2-}$ & 873.32735 & 0.40369 & 0.0 \\
$\mathrm{U}^{4+}-\mathrm{U}^{4+}$ & 0.0 & 0.0 & 0.0 \\
\hline \hline
\end{tabular}

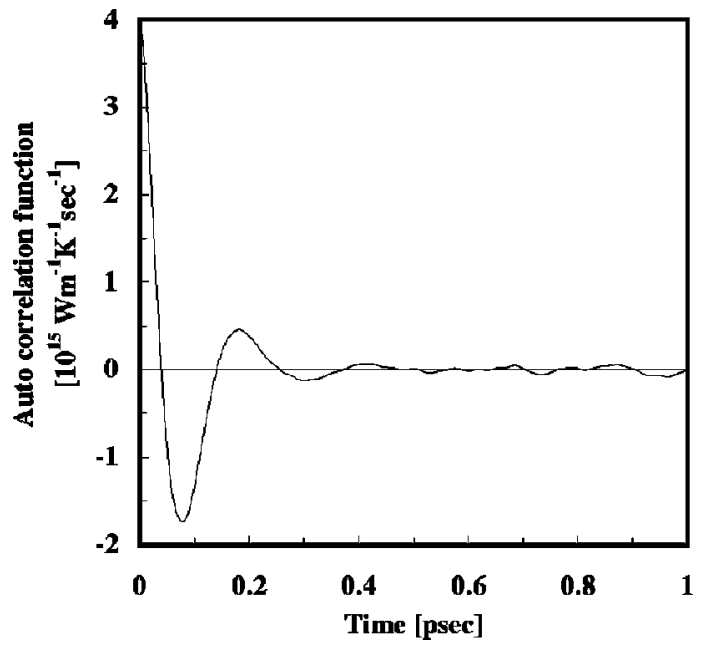

FIG. 5. Autocorrelation function in $\mathrm{UO}_{2}$-EMD calculation.

$$
\begin{aligned}
& \times\left(\cos \left(2 \pi \mathbf{h} \cdot \mathbf{r}_{\mathbf{i}}\right) \sum_{j=1}^{N} Z_{j} \sin \left(2 \pi \mathbf{h} \cdot \mathbf{r}_{\mathbf{j}}\right)\right. \\
& \left.+\sin \left(2 \pi \mathbf{h} \cdot \mathbf{r}_{\mathbf{i}}\right) \sum_{j=1}^{N} Z_{j} \cos \left(2 \pi \mathbf{h} \cdot \mathbf{r}_{\mathbf{j}}\right)\right) .
\end{aligned}
$$

The conditions of the equilibrium molecular dynamics simulation of $\mathrm{UO}_{2}$ are number of particles, 1,500; lattice constant, ${ }^{13,15} 5.58868806 \AA$; total simulation steps, 200000 ; unit time step, $1.0 \mathrm{fsec}$; algorithm, Gear (6 value 1st order); temperature, $2100 \mathrm{~K}$. The system size was determined by the accuracy of the Ewald summation. And there is no significant dependence of the system size in our results when $\mathrm{N}=$ 768.

We show the autocorrelation function of the energy current $1 / V k_{B} T^{2}\langle\mathbf{J}(s) \mathbf{J}(0)\rangle$ in Fig. 5 and the integral of the autocorrelation function $\lambda(t)$ in Fig. 6. From Fig. 5 we see a significant oscillatory behavior in the auto correlation function of $\mathrm{UO}_{2}$, which indicates a remarkable contrast with that of Ar. This oscillation continues to about $10^{-12} \mathrm{sec}$. There-

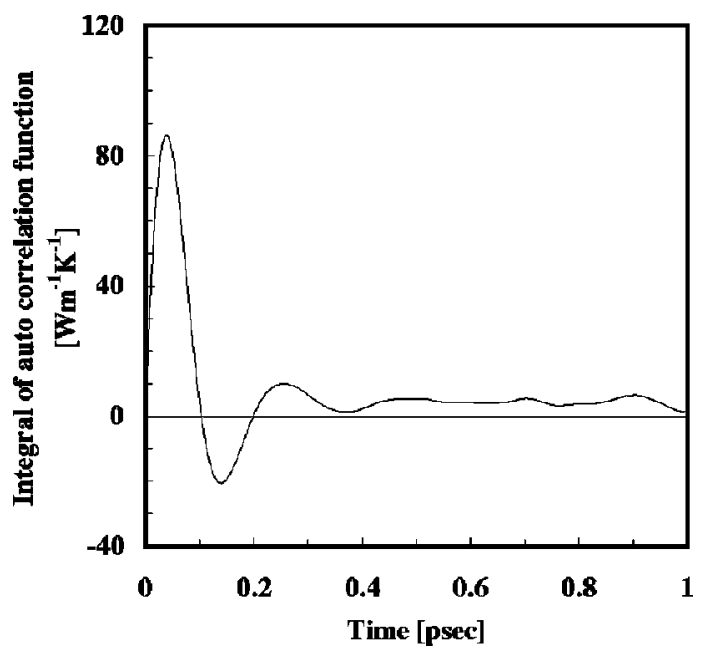

FIG. 6. Integral of autocorrelation function in $\mathrm{UO}_{2}$-EMD calculation. 

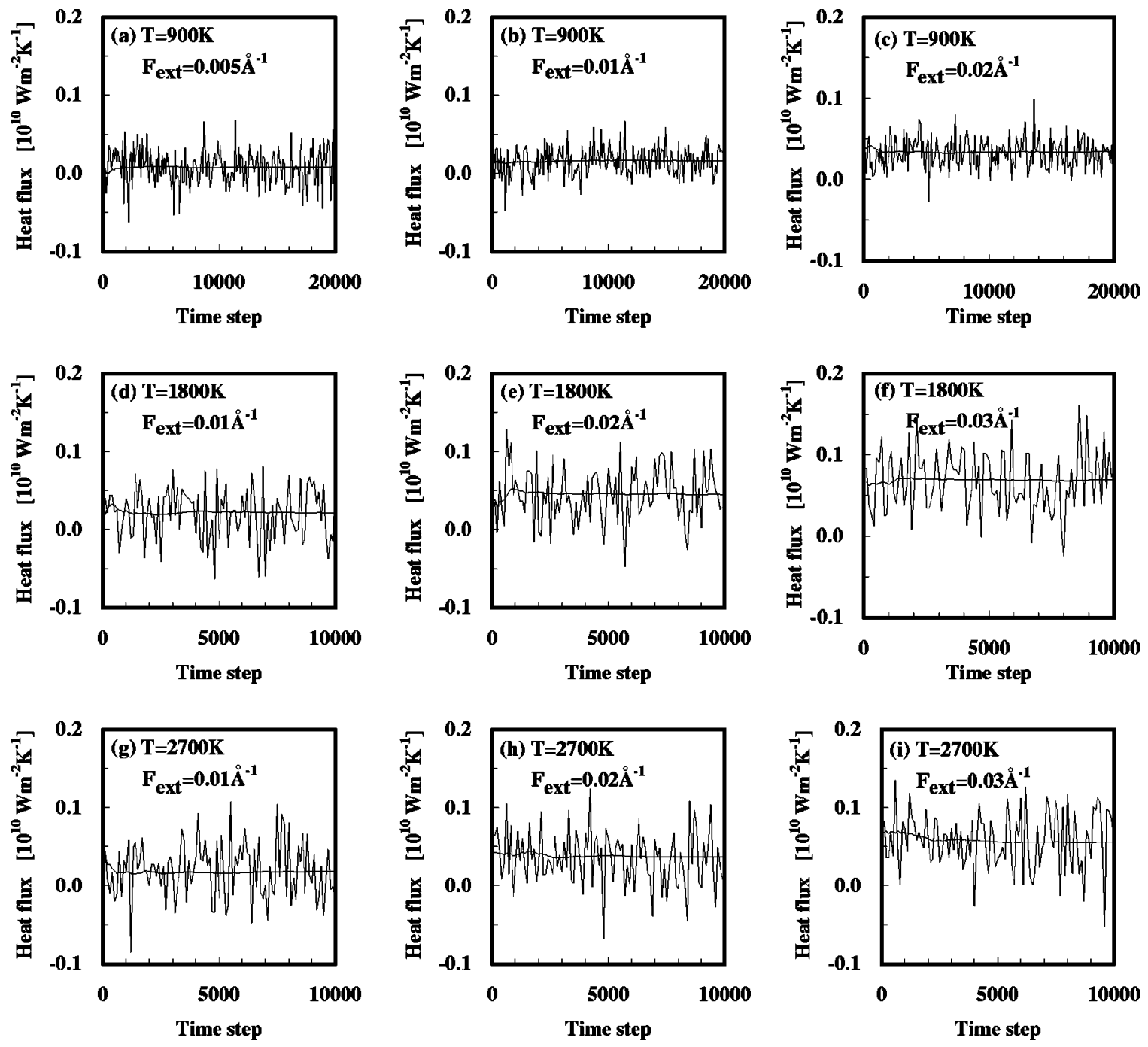

FIG. 7. Heat fluxes as a function of time step. (a) $T=900 \mathrm{~K}, F_{\text {ext }}=0.005 \AA^{-1}$; (b) $T=900 \mathrm{~K}, F_{\text {ext }}=0.01 \AA^{-1}$; (c) $T=900 \mathrm{~K}, F_{\text {ext }}$ $=0.02 \AA^{-1}$; (d) $T=1800 \mathrm{~K}, F_{\text {ext }}=0.01 \AA^{-1}$; (e) $T=1800 \mathrm{~K}, F_{\text {ext }}=0.02 \AA^{-1}$; (f) $T=1800 \mathrm{~K}, F_{\text {ext }}=0.03 \AA^{-1}$; (g) $T=2700 \mathrm{~K}, F_{\text {ext }}$ $=0.01 \AA^{-1}$; (h) $T=2700 \mathrm{~K}, F_{\text {ext }}=0.02 \AA^{-1}$; (i) $T=2700 \mathrm{~K}, F_{\text {ext }}=0.03 \AA^{-1}$.

fore simulation over several 100000 steps is needed to calculate the autocorrelation function. Therefore it is actually difficult to calculate the thermal conductivity through the equilibrium molecular dynamics simulation. So, it becomes advantageous to use the nonequilibrium molecular dynamics simulation for Coulomb systems, as we will show below.

\section{Nonequilibrium molecular dynamics in $\mathrm{UO}_{2}$}

In Coulomb systems, we set $\overleftrightarrow{\mathbf{D}}_{\mathbf{i}}$ as follows: ${ }^{4,5,14}$

$$
\overleftrightarrow{\mathbf{D}}_{\mathbf{i}}=\overleftrightarrow{\mathbf{D}}_{\mathbf{i}}^{*}-\frac{1}{N} \sum_{j=1}^{N} \overleftrightarrow{\mathbf{D}}_{\mathbf{j}}^{*},
$$

where

$$
\begin{aligned}
\stackrel{\mathbf{D}}{\mathbf{i}}^{*}= & {\left[\frac{\left|\mathbf{p}_{\mathbf{i}}\right|^{2}}{2 m_{i}}+\Phi\left(\left|\mathbf{r}_{\mathbf{i}}\right|\right)\right]\left(\begin{array}{ccc}
1 & 0 & 0 \\
0 & 1 & 0 \\
0 & 0 & 1
\end{array}\right) } \\
& +\frac{1}{4 \pi \epsilon_{0}}\left(\begin{array}{ccc}
S_{x x} & S_{y x} & S_{z x} \\
S_{x y} & S_{y y} & S_{z y} \\
S_{x z} & S_{y z} & S_{z z}
\end{array}\right)
\end{aligned}
$$

and $S_{a b}$ are the same as in Eq. (32). At the presence of $\alpha$ introduced in Eq. (2), when the system temperature has been changed from a desired temperature by unexpected numerical errors, it is not always possible to return to the desired temperature. Instead, we have used the method of Nosé and Hoover thermostat control. ${ }^{16,17}$

The conditions of nonequilibrium molecular dynamics simulation of $\mathrm{UO}_{2}$ are number of particles, 1500; total simulation steps, 20000 from 300 to $900 \mathrm{~K}$, and 10000 from 1200 to $3000 \mathrm{~K}$; unit time step, $1.0 \mathrm{fsec}$; algorithm, Gear (6 value 1st order); temperature, from 300 to $3000 \mathrm{~K}$. We have used the lattice constant $L$ at each temperature obtained by $^{13,15}$

$$
\begin{aligned}
L= & 5.47 \times 10^{-10}\left(0.997+1.0 \times 10^{-5} T\right. \\
& \left.+4.0 \times 10^{2} e^{-6.9 \times 10^{-20} / k_{B} T}\right)(\mathrm{m}) .
\end{aligned}
$$

In Fig. 7 we show the heat flux $\psi$ and the average of the heat flux $\bar{\psi}$ as a function of time step in 900, 1800, and 2700 $\mathrm{K}$. We have calculated the average of heat flux $\bar{\psi}$ with instanteneous $\psi$ 's at $t=t_{i}$ as follows: 


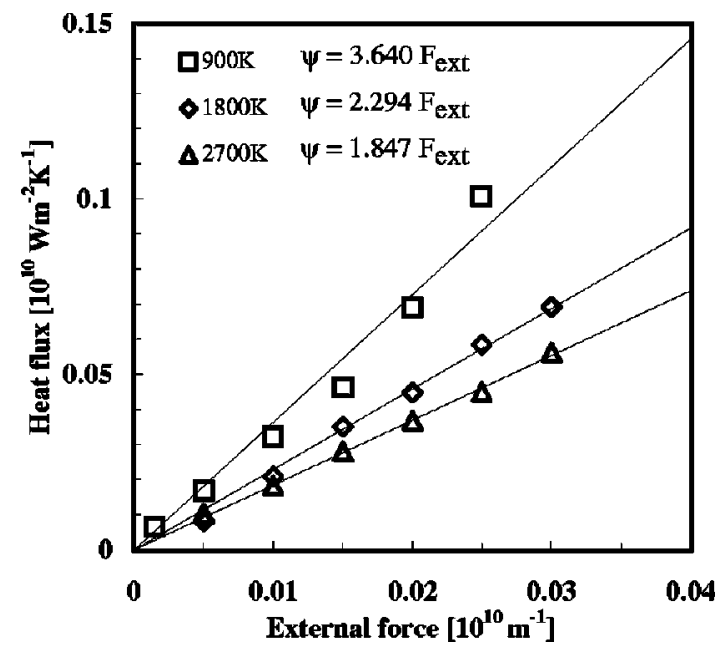

FIG. 8. Heat fluxes as a function of the external force in $\mathrm{UO}_{2}$-NEMD calculation.

$$
\bar{\psi}=\left.\frac{1}{N} \sum_{i=1}^{N} \psi\right|_{t=t_{i}}
$$

From Fig. 7, $\psi$ (gray line) is intensively changed according to $t$, but the average $\bar{\psi}$ (black line) converges to a fixed value.

We show the relation between $F_{\text {ext }}$ and $\psi\left(F_{\text {ext }}\right)$ by the present nonequilibrium molecular dynamics calculation in Fig. 8. The straight lines in Fig. 8 are obtained by leastsquare fitting of $F_{\text {ext }}$ and $\psi\left(F_{\text {ext }}\right)$ at each temperature. The values of the thermal conductivity at 900,1800 , and $2700 \mathrm{~K}$ are obtained 3.747, 2.294, and $1.862 \mathrm{~W} \mathrm{~m}^{-1} \mathrm{~K}^{-1}$. In Fig. 9 and Table II we show the thermal conductivity obtained by the present molecular dynamics calculation together with experiments. $^{12,13}$

\section{DISCUSSIONS}

\section{A. Thermal conductivity of Ar}

Thermal conductivity of Ar obtained by nonequilibrium molecular dynamics calculation at temperatures from $40 \mathrm{~K}$ up to $80 \mathrm{~K}$ shows the same tendency as the experimental

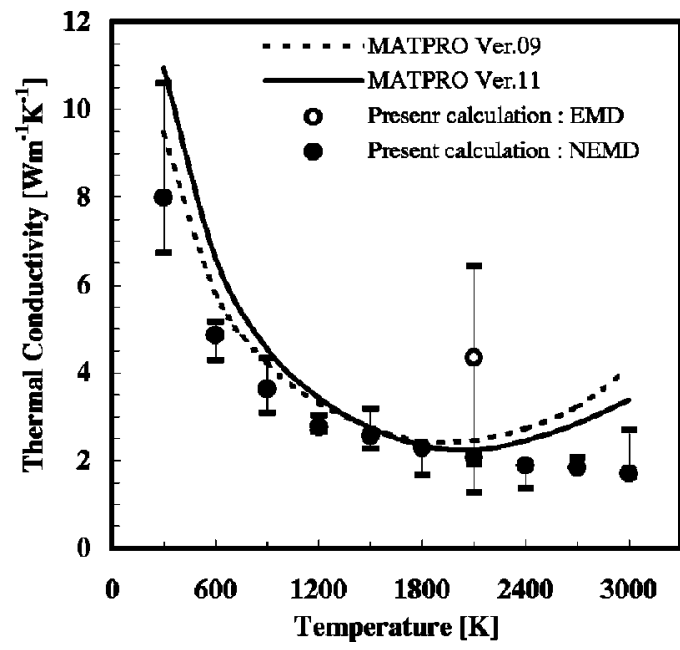

FIG. 9. Thermal conductivity of $\mathrm{UO}_{2}$.
TABLE II. Thermal conductivity of $\mathrm{UO}_{2}$.

\begin{tabular}{cc}
\hline \hline Temperature $[\mathrm{K}]$ & Thermal conductivity $\left[\mathrm{W} \mathrm{m}^{-1} \mathrm{~K}^{-1}\right]$ \\
\hline 300 & 7.991 \\
600 & 4.864 \\
900 & 3.640 \\
1200 & 2.768 \\
1500 & 2.567 \\
1800 & 2.294 \\
2100 & 2.073 \\
2400 & 1.891 \\
2700 & 1.847 \\
3000 & 1.718 \\
\hline
\end{tabular}

behavior. However, the thermal conductivity with the same lattice constant in this temperature range yields values smaller than experimental ones.

For Ar, the same values were also obtained using the equilibrium molecular dynamics simulation. It is concluded that the evaluation of the thermal conductivity by the nonequilibrium molecular dynamics simulation is quite useful.

\section{B. Thermal conductivity of $\mathrm{UO}_{2}$}

We have calculated the thermal conductivity of $\mathrm{UO}_{2}$ at temperatures from $300 \mathrm{~K}$ up to $3000 \mathrm{~K}$ by the nonequilibrium molecular dynamics simulation. At the range of temperature from $1200-2100 \mathrm{~K}$, the result was in good agreement with experiments. However, at other temperature range, the same agreement between calculation and experiment is not always as good as above.

Above $2400 \mathrm{~K}$, the thermal conductivity (experiments) shows an increase as the temperature increases because of the contribution of the electron conduction. In the present calculation, we did not take into consideration such effects. Below $1200 \mathrm{~K}$, a reasonable agreement was obtained between the present result and experiments. But the absolute value of calculation is a little smaller than experiments. A partial reason for this is considered by the conditions used in this calculation that the external force are not small enough to estimate a correct linear relation between $F_{\text {ext }}$ and $\psi$. On the other hand, using a weak external force, the oscillatary behavior of the heat flux becomes stronger. Thus in order to obtain an accurate thermal conductivity, many simulation steps are necessary. At low temperatures, simulation steps in the present calculation may not be enough.

\section{Melting point of $\mathrm{UO}_{2}$}

We show the mean-square displacement at several temperatures in Fig. 10. It is seen that $\mathrm{O}$ (oxide) atoms are diffusive, while U (uranium) atoms are not. Such structural nature is similar to that of the solid electrolytes. We have also calculated the mean-square displacement at higher temperatures, and tried to find the temperature where the frame of $U$ is collapsed (melted). If we estimate the melting temperature by the collapse of the frame, it is about $1000 \mathrm{~K}$ higher than the experiment one. Since the potential parameters used in this work are fitted at $300 \mathrm{~K}$, they may not be good for the estimation of the melting point where the lattice expansion 

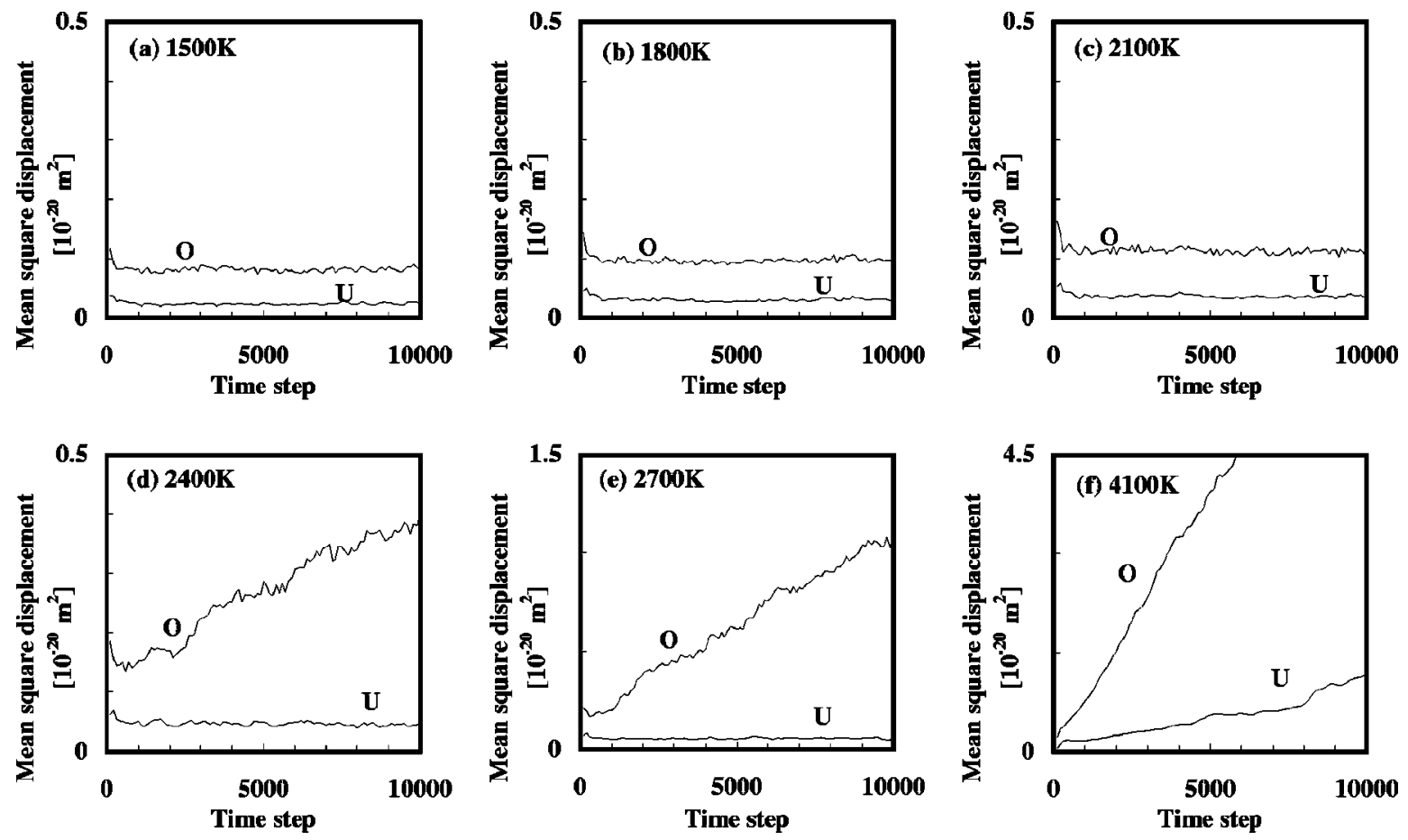

FIG. 10. Mean-square displacement of $\mathrm{UO}_{2}$. (a) $T=1500 \mathrm{~K}$; (b) $T=1800 \mathrm{~K}$; (c) $T=2100 \mathrm{~K}$; (d) $T=2400 \mathrm{~K}$; (e) $T=2700 \mathrm{~K}$; (f) $T$ $=4100 \mathrm{~K}$.

may be considerable. In addition, an over heating property becomes important especially for a small system as used in the present work.

\section{Others}

The present work is nothing but a first step to discuss the thermal conductivity of MOX by molecular dynamics simulation. In this work, we have succeeded in evaluating $\lambda$ of $\mathrm{UO}_{2}$. As a next step, we are examining other physical quantities such as elastic constants and the heat capacity of $\mathrm{UO}_{2}$, which will be reported elsewhere. We are also interested in how to construct a better model for $\mathrm{UO}_{2}$ and MOX. It is confirmed that the thermal expansion coefficient of $\mathrm{UO}_{2}$ obtained by using the present potential model is not in good agreement with experiments. ${ }^{18}$ And we want to clear the reason why the thermal conductivity of the MOX pellet is a little smaller than that of $\mathrm{UO}_{2}$. We infer the reason that the MOX system has some amorphous characteristic because of $\mathrm{Pu}$ atom is not placed periodically. ${ }^{19}$

\section{ACKNOWLEDGMENTS}

We would like to express our sincere thanks to Dr. Giovanni Ciccotti and Dr. Carlo Pierleoni for suggesting to us a part of the calculation techniques used in this work.
${ }^{1}$ P.B. Allen and J.L. Feldman, Phys. Rev. B 48, 12581 (1993).

${ }^{2}$ D.J. Evans and G.P. Morriss, Statistical Mechanics of Nonequilibrium Liquids (Academic, New York, 1990).

${ }^{3}$ Astuhiko Maeda and Toyonori Munakata, Phys. Rev. E 52, 234 (1995).

${ }^{4}$ C. Pierleoni, G. Ciccotti, and B. Bernu, Europhys. Lett. 4, 1115 (1987).

${ }^{5}$ C. Pierleoni and G. Ciccotti, J. Phys.: Condens. Matter 2, 1315 (1990).

${ }^{6}$ M.P. Allen and D.J. Tildesley, Computer Simulation of Liquids (Oxford Science Publications, New York, 1987).

${ }^{7}$ J.P. Hansen and I.R. McDonald, Theory of Simple Liquids, 2nd ed. (Academic, New York, 1986).

${ }^{8}$ David K. Christen and Gerald L. Pollack, Phys. Rev. B 12, 3380 (1975).

${ }^{9}$ F. Clayton and D.N. Batchelder, J. Phys. C 6, 1213 (1973).
${ }^{10}$ C.R.A. Catlow, Proc. R. Soc. London, Ser. A 353, 533 (1977).

${ }^{11}$ J.R. Walker and C.R.A. Catlow, J. Phys. C 14, 979 (1981).

${ }^{12}$ EG\&G Idaho, Inc., MATPRO Ver. 09 a Handbook of Materials Properties for Use in the Analysis of Light Water Reactor Fuel Rod Behavior, Appendix A, TREE-NUREG-1005 (1976).

${ }^{13}$ EG\&G Idaho, Inc., MATPRO Ver. 11 Rev. 2 a Handbook of Materials Properties for Use in the Analysis of Light Water Reactor Fuel Rod Behavior, Appendix A, NUREG/CR-0497, TREE1280, Rev. 2 (1981).

${ }^{14}$ B. Bernu and P. Vieillefosse, Phys. Rev. A 18, 2345 (1978).

${ }^{15}$ B.F. Rubin, AEC Research and Development, Report No. GEAP13582, 1970 (unpublished).

${ }^{16}$ S. Nosé and M.L. Klein, Mol. Phys. 50, 1055 (1983).

${ }^{17}$ S. Nosé, Mol. Phys. 52, 255 (1984).

${ }^{18}$ R. Okawa and H. Kimizuka (private communication).

${ }^{19}$ J. Michalski, Phys. Rev. B 45, 7054 (1992). 